hiesiger Gegend sahe ich die schwarzen Störche früher als die weissen, ja, manche der letzten kamen sechs Wochen später, als gewöhnlich. Zwei Schiffs-Capitaine hiesiger Gegend, welche sich Ende März im mittelländischen Meere auf der Höhe von Malta befanden, berichteten:

Dass bei einem plötzlich ausgebrochenen Sturme die Schiffe so von Störchen bedeckt wurden, dass dadurch Gefahr für die Schiffe befürchtet wurde, und dass sie mehrere Tage darnach zwischen auf dem Meere schwimmenden Störchen gesegelt. Es ist dadurch eine sehr grosse Menge zu Tode gekommen und von den überlebenden eine bedeutende Anzahl erschlagen, so dass sie spät oder überhaupt nicht an den Brutplätzen angekommen sind. Es scheint dies Unwetter die später ziehenden nördlichen Störche besonders getroffen zu haben, denn in der Maingegend sollen die Störche zur gewöhnlichen Zeit und in gewöhnlichen Verhältnissen eingetroffen sein.

Warbelow bei Stolp.

Eug. v. Homeyer.

\title{
Einige ornithologische Notizen, gesammelt auf einer Reise durch Kroatien, Dalmatien und Montenegro.
}

Von

Anton Fritsch, in Prag.

Wenn es schon bei uns in Böhmen schwer fällt, der Ornithologie treu zu bleiben, da alle übrigen Naturerzeugnisse, und besonders die prachtvollen Petrefacten sehr zu ihrem Studium einladen, so erfordert es gewiss noch mehr Standhaftigkeit die liebe Vogelwelt nicht ganz zu vergessen, sobald man die Schätze des reichbelebten Mittelmeers auf einem Fischmarkte angehäuft erblickt. Man sieht hier auch zwar Geschöpfe, welche Flügel haben und auch Namen von Vögeln tragen, (Trigla corvus, Tr. corax, Tr. hirundo etc.) doch sind es eben keine Vögel.

Obgleich ich diese Reise um Seethiere zu beobachten und einzusammeln, unternahm, ging ich doch nie ohne mein Gewehr von Hause und unterliess nicht auf allen meinen Excursionen die hier sparsame Vogelwelt zu beobachten.

Am 2. März. In Fiune angekommen, sah ich im Hafen einige Dutzend Möven, unter denen Larus minutus mit seinem schwarzen Köpfchen am kenntlichsten war. Die übrigen durchaus grossen Arten schienen Larus glaucus, marinus und argentatus zu sein. Die Zahl der Möven ist hier auffallend gering und wenn das Meer ruhig ist, so verschwinden nach und nach alle aus dem Hafen, um erst bei stürmischem Welter 
zurückzukehren. Sterna-Arten bemerkte ich diesmal keine, aber als ich einmal später, Anfangs Mai, wieder nach Fiume kam, hielt sich in der Nähe des Ufers ein grosser Schwarm von Sterna nigra auf. Die jungen Fiumaner machten sich ein Vergnügen daraus nach denselben mit Steinen zu werfen und trafen wirklich einige im Fluge, wozu doch gewiss viel Gewandtheit gehört.

Von Fiume begab ich ich mich am 3. März nach Bribir, einer Stadt im Vinodoler Thale, die auf längere Zeit mein Standquartier wurde. Mein hier ansässiger Freund, der Forstverwalter Gröger, hatte ein jebendes Steinhuhn (kroatisch Orebica genannt) hinter dem Fenster, welches aber sehr ungestüm und wild sich zeigte, obgleich es schon ein halbes Jahr lang in der Gefangenschaft war.

Auf der ersten Excursion die ich am 4. März unternahm, fiel mir sogleich der zirrende Gesang eines Ammerlings auf, welchen ich alsbald erlegte, und in ihm ein hübsches Männchen von Emberiza cirlus erkannte. Diese Vogelart ist hier eine der häufigsten, sie sitzt meist auf den Gipfeln von Obstbäumen, welche in den hier ungemein zahlreichen Weingärten einzeln stehen. An diesem Tage beobachtete ich noch: Motacilla sulphurea, Rubecula rubecula, Turdus musicus, einige ParusArten und Scolopax gallinago.

Den 6. März gingen wir mit meinem Freunde auf Steinhühner aus, und nachdem wir landeinwärts eine Stunde bergauf gestiegen waren, erreichten wir ein Terrain, welches gleichsam die erste Stufe des noch höher sich erhebenden Gebirges war. Der Boden war hier felsig, rissig und mit dornigem Gestripp bewachsen, in welchem wir einige Ketten Steinhühner antrafen. Die Steinhühner fliegen hier nicht so direkt auf, wie unsere Hühner und Fasanen, sondern brauchen immer 4-5 Secunden, bevor sie sich aus dem Gestripp erheben können, wodurch es dem Jäger möglich ist, sich schussbereit zu machen. Bei dem gefährlichen Springen von einer Felsenplatte zur andern, und bei dem Winden durch das an den Kleidern immerwährend zerrende Dorngestripp würde ein Erlegen von Steinhühnern in dieser Gegend fast unmöglich sein.

Der Beschreibung nach zu urtheilen, wurde hier im vergangenen Herbste ein Exemplar von Perdix rubra hoch oben im Gebirge geschossen.

Wenn der sehr gefürchtete südöstliche Wind „Bora" die Berge unersteigbar macht, so ziehen sich auch die Steinhühner von der Höhe herab. Am nächsten Tage, den 7. März, traf ich einige ganz nahe beim Meere, als ich ausging, um eine Grotte bei Novi zu untersuchen. Diesen Tag sah ich Saxicola rubicola, Machetes pugnax, Vanellus cristatus; Columba livia strich pfeilschnell mit dem heftigen Winde kämpfend am 
Meeresufer hin und Eistaucher (Colymbus arcticus?) und einige Enten hielten sich in der Nähe des Ufers auf, um hinter den Felsenblöcken Schutz vor der Bora zu finden.

Am 15. sah ich im Vinodoler Thale Alauda arborea, Anthus pratensis, Saxicola rubicola und ein Lugar (Waldheger), erlegte Anas strepera, nyroca und Querquedula.

Vom 14. bis zum 17. dauerte die Bora mit ungewöhnlicher Heftigkeit fort und meine Beobachtungen wurden darauf beschränkt, durch ein Jalousie-Fenster in den kleinen Garten zu sehen, der an unserem Wohngebäude lag und durch dasselbe vor dem unmittelbaren Einfluss des Sturmwindes geschützt wurde.

Da versammelte sich eine grosse Menge von Misteldrosseln, Turdus viscivorus, und Singdrosseln T. musicus, und suchten fleissig nach Nahrung in dem Epheu mit dem die Wände dicht bewachsen waren. In diesem Epheu herrschte als König ein Rothkehlchen, welches mit einem kleinen schwarzköpfigen Sänger (Sylvia atricapilla? oder melanocephala) in ununterbrochenem Kampfe lebte, und ihn aus seinem Reviere $z u$ vertreiben suchte.

Am 19. schoss ich einige Scolopax gallinago und am 20. wurde mir ein Männcheu von Tichodroma muraria überbracht, das auf einem über dem Meere stehenden Hause in Cirkuenica geschossen wurde. Denselben Tag erhielt ich auch Ruticilla erithaca.

Während ich nun bis zum 28. täglich den Meeresstrand zwischen Novi, Selce und Cirkuenica besuchte, war mir wirklich die Armuth in der Vogelwelt auffallend. Die ganze Zeit hindurch sahe ich blos ein Mövenpaar, einige Eistaucher und ein Pärchen von Corvus Corax, welches auf den steilen Felsenufern sein Nest hatte und den Ufern entlang fleissig nach den vom Meere ausgeworfenen Thieren suchte.

Emberiza cirlus und die Singdrosseln waren die einzigen steten Sänger, welche mich des frühen Morgens auf meinen Wanderungen begrüssten.

Ungeduldig über die Einförmigkeit, welche nach einiger Zeit auch in meinen Acquisitionen von Seethieren sich zeigte, verliess ich Croatien und hoffte im Süden bessere Beute zu machen.

Am 31. trat ich meine Weiterreise an, mit der Absicht, bis nach Cattaro mich zu begeben, wo ich auch am 7. April eintraf. Auf der ganzen Reise habe ich keinen Vogel wahrgenommen, ausser einer alten Weihe (Circus pallidus?), welche sich zeitlich früh in der Nähe unseres Dampfers zeigte, eben als wir bei Ragusa nahe daran waren, von einem Siroko-Sturme an das Land geschleudert zu werden. 
In Cattaro selbst kaufte ich auf der Strasse 3 Stück Numenius tenuirostris, welche zugleich mit Columba oenas zum Braten bestimmt waren. Ein hiesiger Kaufmann kaufte um $6 \mathrm{Kr}$. $=2 \mathrm{Sgr}$. ein schönes Exemplar von Ardea egretta, an welchem ich ihm das Ausstopfen zeigte. Ich konnte nicht erfahren, woher diese Sumpfvögel gebracht wurden, vermuthe aber, dass aus der Bocca von Cattaro selbst, weil ich auf der Rückreise am Ufer des Meeres 12 Stück Ardea comata erblickte, welche aber gewiss nur auf dem Zuge waren, da ihnen die hierortigen Localitäten keinen bleibenden Wohnsitz darbieten.

Am 10. April trat ich die Reise nach Montenegro an und als ich auf dem berühmten Zigzagwege emporstieg, wurde ich auf das ungestüme Geschrei eines Vogels aufmerksam, das ich nie früher gehört hatte. Der Vogel entzog sich lange einer näheren Beobachtung, bis er endlich nahe am Wege sich zeigte und als Sitta syriaca Ehrbg zu erkennen gab.

Während unsere Sitta caesia einzig die Bäume von Ungeziefer reinigt, treibt sich die $S$. syriaca auf den kahlen Felsen umher und es ist kein Wunder, wenn sie als Gebirgsbewohner sowohl am Körper, als auch an der Stimme stärker ist.

Am Plateau des Montenegrinischen Berges angelangt, sah ich auf den kleinen Feldern, welche zwischen den kahlen Felsen sich befinden, viele Saxicola stapazina.

Sie waren wenig scheu und erhoben sich singend einige Schuh von der Erde, auf welche sie sich nach kurzem Flattern wieder herabliessen. Ein Accentor alpinus flog an mir vorbei, und verschwand in eine Felsenspalte-in der ich sein nosh leeres Nest fand.

Am Abende in Celtine, der Hauptstadt von Montenegro angelangt, bemerkte ich in der Dämmerung einen Edelfalken - den ersten auf der ganzen Reise - und doch ging ich in der Hoffnung nach Dalmatien Falco concolor, Eleonorae, Feldeggii u. a. zu sehen!

Die Vogelwelt von Montenegro fand ich viel mannigfaltiger, als ich den 13. am Flusse Rjeka nach dem Scutari-See ruderte.

Die Ufer des Flusses werden nach und nach sumpfig, bis man ohne es zu bemerken auf dem Scutari-See selbst sich befindet. Auf dieser interessanten Fahrt sah ich 12 Stück Pelecanus crispus, eine Schaar Carbo pygmaeus, Ardea garzetta und cinerea. Die Ufer des Sees sind gegen die Mońtenegrinische Cenická Nahia zu, sumpfig und mit viel Weidengestripp bewachsen und dürften Gelegenheil zu manchen interessanten Beobachtungen geben, aber meine Zeit erlaubte mir nicht, hier länger zu bleiben. Es wäre auch aus Sicherheitsrücksichten nicht 
rathsam sich in dieser Gegend durch wiederholtes Schiessen bemerkbar zu machen, weil es eben der Ort ist, wo die häufigsten Zusammenstosse mit den Türken vorkommen und man sehr leicht für einen Spion gehalten und erschossen werden könnte. Am Nachmittage des năchsten Tages stieg ich wieder von den Montenegrinischen Bergen gegen die östreichische Festung Budua herab. In einem Olivenwäldchen angelangt, erlegte ich mit dem Stockgewehre einige Laubvögel in der sicheren Hoffnung, es werde Phyllopneuste olivetorum sein und da es nichts weiter als $P$. sibilatrix war, so ging mir das gar nicht in den Kopf, dass auf einem Olivenbaume etwas Anderes als $P h$. olivetorum vorkommen könne.

Ich bin wirklich in Verlegenheit, was ich über die Vogelarten der Insel Lesina sagen soll, da mir doch während meines dreiwöchentlichen Aufenthaltes daselbst, ausser einigen Hänflingen und Stieglitzen kaum ein anderer lebender Vogel zu Gesichte kam. Auf einer Excursion zur See sah ich Puffinus cinereus und auf der Insel Forcola ein CircusPärchen, Saxicola rubetra und eine Larus-Colonie. Die Fischer brachten mir 3 junge Corvus corax.

Einige Thäler mit üppiger Vegetation, durch welche ich von der Stadt Lesina nach der Stadt Citta veccia ging, schienen geeignet zu sein, kleinere Sänger zu beherbergen; doch erblickte ich keinen und hörte nur von einer Felsenwand herab das Geschrei junger Falken; auch sah ich einige Stücke von Fringilla serinus $L$.

Durch das Unglück einer Fussverrenkung, wurde meine weitere Thätigkeit fast gleich Null gesetzt. Als ich später einige Excursionen in Croatien machte, bemerkte ich an den steilen Meeresufern die blaue Steindrossel. Im Vinodoler Thale hatten sich Euspiza melanocephala eingefunden, im übrigen blieb die Armuth doch sehr auffallend.

Zum Schlusse muss ich bemerken, dass die Fundgrube für die Ornithologie in Dalmatien Narenta ist, woselbst auch der Oberst, Baron Feldegg, viele schöne Arten einsammelte, welche ich aus seiner Sammlung für unser Museum einkaufte, und in meinem Werke über Vögel Europa's abbilden lasse. Ich konnte diese Gegend nicht besuchen da die ornithologischen Beobachtungen diesmal nur ein Nebenzweck' waren. 


\section{$2 \mathrm{BHL}$ Biodiversity Heritage Library}

1858. "Einige ornithologische Notizen, gesammelt auf einer Reise durch Kroatien, Dalmatien und Montenegro." Journal $f u$

r Ornithologie 6, 411-415. https://doi.org/10.1007/bf02010069.

View This Item Online: $\underline{\text { https://www.biodiversitylibrary.org/item/49379 }}$

DOI: https://doi.org/10.1007/bf02010069

Permalink: https://www.biodiversitylibrary.org/partpdf/141372

\section{Holding Institution}

Natural History Museum Library, London

\section{Sponsored by}

Natural History Museum Library, London

\section{Copyright \& Reuse}

Copyright Status: Public domain. The BHL considers that this work is no longer under copyright protection.

This document was created from content at the Biodiversity Heritage Library, the world's largest open access digital library for biodiversity literature and archives. Visit BHL at https://www.biodiversitylibrary.org. 